\title{
Topological order parameters for interacting topological insulators
}

\author{
Zhong Wang, ${ }^{1,2}$ Xiao-Liang Qi, ${ }^{3,2}$ and Shou-Cheng Zhang ${ }^{2}$ \\ ${ }^{1}$ Department of Modern Physics, University of Science and Technology of China, Hefei, 230026, P. R. China \\ ${ }^{2}$ Department of Physics, McCullough Building, Stanford University, Stanford CA 94305-4045 \\ ${ }^{3}$ Microsoft Research, Station Q, Elings Hall, University of California, Santa Barbara, CA 93106
}

\begin{abstract}
We propose a topological order parameter for interacting topological insulators, expressed in terms of the full Green's functions of the interacting system. We show that it is exactly quantized for a time reversal invariant topological insulator, and it can be experimentally measured through the topological magneto-electric effect. This topological order parameter can be applied to both interacting and disordered systems, and used for determining their phase diagrams.
\end{abstract}

PACS numbers: 73.43.-f,71.70.Ej,75.70.Tj

Recently, topological insulators(TI) have attracted great attention in condensed matter physics[1-3]. Historically, the concept of the time reversal $(\mathcal{T})$ invariant TI has been developed along two independent routes[1]. The topological field theory (TFT) approach was first introduced by Zhang and $\mathrm{Hu}[4]$, who constructed a microscopic model of the $\mathcal{T}$ invariant TI in four spatial dimensions $(4+1 \mathrm{~d})$, and showed that the effective TFT is described by the $4+1 \mathrm{~d}$ ChernSimons(CS) action[5]. In contrast to the $\mathcal{T}$ breaking CS action in $2+1 d$, the CS action in $4+1 d$ preserves the $\mathcal{T}$ symmetry. It is now understood that this state is the fundamental $\mathcal{T}$-invariant TI state, from which both the three and the two dimensional $\mathcal{T}$-invariant TIs can be derived[6-8]. Using the simple procedure of dimensional reduction, the TFT for three and two dimensional $\mathcal{T}$-invariant TIs has been constructed[6]. Independently, the topological band theory (TBT) was first developed starting from the pioneering work of Kane and Mele [9], who first proposed a $Z_{2}$ topological invariant within the non-interacting band theory. This non-interacting $Z_{2}$ topological invariant has been generalized to the three dimensional TIs 10-12]. The TFT is generally valid for systems with interactions and disorder; recently, it has been shown that the TFT reduces exactly to the the TBT in the non-interacting limit[13]. The TFT has been further developed recently[1417.

TIs are widely believed to be a new state of quantum matter. Since all states of matter in Nature, including band insulators, are necessarily interacting, it is important to formulate a general definition of TIs which is valid for general interactions and disorder. This is especially important since an explicit counter-example in one dimension has been constructed where a non-interacting topological state becomes unstable against interactions[18]. After the theoretical prediction and experimental discoveries of the weakly interacting TIs [1-3], it would be most interesting to investigate topological Mott insulators where the interaction plays an essential role [19-26]. In the case where strong electronic correlations play a crucial role, it is essential to define a general topological order parameter which can determine the phase diagram of these systems.

The TFT approach defines the generally interacting TI in terms of a topological term in the effective electromagnetic action. The $\theta$ angle, or equivalently the electromagnetic po-

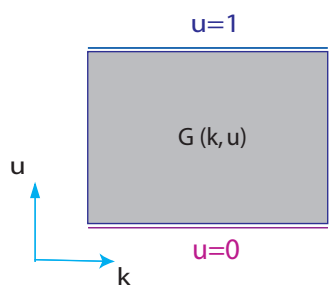

(a)

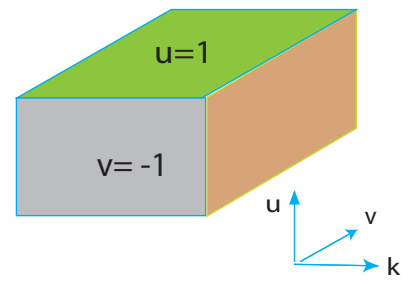

(b)
FIG. 1: Extension of $G(k)$ to higher dimensional manifolds $(k, u)$ and $(k, u, v)$. (a)Extension of $G(k)$ to $G(k, u), u \in[0,1]$ in the definition of $P_{3}$, with $G(k, u=0)=G(k)$. The $3+1 \mathrm{~d}$ momenta space is illustrated as horizontal 1d interval. (b)Extension of $G(k)$ to $G(k, u, v)$ with $G(k, u=v=0)=G(k)$ in the definition of $P_{2}$.

larization $P_{3}$, can only take two discrete values in a system with $\mathcal{T}$ symmetry. In this work, we give an explicit formula of $P_{3}$ which can be evaluated in a generally interacting system. Our explicit formula for the topological order parameter $P_{3}$ satisfies the following general criteria: (1) It is well defined in the presence of interaction and disorder. (2) It takes quantized values, invariant against small changes of parameters in the model Hamiltonian or in experiment. (3) It is experimentally measurable. The TBT is based on the single particle band states, and can not be easily generalized to interacting systems. On the other hand, the TFT is valid for systems with general interactions and disroder, and the quantized magneto-electric polarization can be directly measured experimentally [6, 14, 27, 28]. The central result of this paper is the topological order parameter of the $3+1 \mathrm{~d}$ TI defined as

$$
\begin{aligned}
P_{3}= & \frac{\pi}{6} \int_{0}^{1} d u \int \frac{d^{4} k}{(2 \pi)^{4}} \operatorname{Tr} \epsilon^{\mu \nu \rho \sigma}\left[G \partial_{\mu} G^{-1} G \partial_{\nu} G^{-1}\right. \\
& \left.\times G \partial_{\rho} G^{-1} G \partial_{\sigma} G^{-1} G \partial_{u} G^{-1}\right]
\end{aligned}
$$

in which $G=G(k, u) ; k=\left(k_{0}, \mathbf{k}\right)$. The momentum variables $\mathbf{k}=\left(k_{1}, k_{2}, k_{3}\right)$ are integrated over the Brillouin zone and frequency $k_{0}$ is integrated over $(-\infty,+\infty) . G(k, u=0) \equiv G(k)$ is the full imaginary time single-particle Green's function of the interacting system, and $G(k, u)$ for $u \neq 0$ is a smooth extension of $G(k, u=0)$ [see Fig.(11)], with a fixed reference value $G(k, u=1)$ corresponding to Green's function of a topo- 
logically trivial insulating state. It is convenient to choose $G(k, u=1)$ as a diagonal matrix with $G_{\alpha \alpha}=\left(i k_{0}-\Delta\right)^{-1}$ for empty bands $\alpha$ and $G_{\beta \beta}=\left(i k_{0}+\Delta\right)^{-1}$ for filled bands $\beta$, where $\Delta>0$ is independent of $\mathbf{k}$. Even though $P_{3}$ is a physical quantity in three dimensions, we introduced a Wess-ZuminoWitten (WZW) [29] type of extension parameter $u$ in its definition. Essentially, we borrowed the ideas of WZW term in coordinate space to the frequency and momentum space. Similar to the WZW term, we shall show that $P_{3}$ is only well-defined modulo an integer, and it can only take quantized value of 0 or $1 / 2$ modulo integer for an $\mathcal{T}$-invariant insulator. The TFT describes the physical response of an insulator in terms of the effective electromagnetic action[6]

$$
\begin{aligned}
S_{\text {eff }} & =S_{\text {Maxwell }}+S_{\text {topo }} \\
& =\int d^{3} x d t\left[\frac{1}{16 \pi} F_{\mu \nu} F^{\mu \nu}+\frac{\theta \alpha}{32 \pi^{2}} \epsilon^{\mu \nu \sigma \tau} F_{\mu \nu} F_{\sigma \tau}\right]
\end{aligned}
$$

where $\theta$ can only take values of 0 or $\pi$ for a $\mathcal{T}$-invariant insulator satisfying periodic boundary condition. We show that the topological order parameter $P_{3}$ enters the physical response function by the identity:

$$
\theta=2 \pi P_{3}
$$

Therefore, our topological order parameter $P_{3}$ satisfies all three main criteria discussed above. Furthermore, the full Green's function entering $P_{3}$ can be directly computed by quantum many-body techniques, such as exact numerical diagonalization, quantum Monte Carlo method, dynamical mean field theory etc.

The fundamental $\mathcal{T}$-invariant TI in $4+1 d$ : Even though we are mostly interested in the $3+1 \mathrm{~d}$ physical space, the presence of the WZW extension parameter $u$ hints that the topological structure of $P_{3}$ is inherited from the $4+1 \mathrm{~d}$ TI [4]. The concept of a $\mathcal{T}$ invariant TI is most naturally formulated in $4+1 \mathrm{~d}$. All the lower dimensional $\mathcal{T}$-invariant TIs can be easily obtained from this fundamental state by a simple procedure of dimensional reduction. For this reason, we discuss the fundamental $\mathcal{T}$-invariant TI first in $4+1 \mathrm{~d}$. The effective TFT for the $4+1 \mathrm{~d}$ $\mathrm{TI}$ is given by a CS term

$$
S_{\text {eff }}=\frac{C_{2}}{24 \pi^{2}} \int d^{4} x d t \epsilon^{\mu \nu \rho \sigma \tau} A_{\mu} \partial_{v} A_{\rho} \partial_{\sigma} A_{\tau}
$$

Under the time reversal transformation, $\mathbf{A} \rightarrow-\mathbf{A}, A_{0} \rightarrow A_{0}$, therefore, we see that this term is explicitly $\mathcal{T}$-invariant.

We first show that $C_{2}$ is quantized to be integer for insulating system without ground state degeneracy on a $4 \mathrm{~d}$ torus $T^{4}=T_{12} \times T_{34}$. Suppose we penetrate a flux quanta $\phi=2 \pi$ into $T_{34}$, with field strength $F_{34}=\partial_{3} A_{4}-\partial_{4} A_{3}=\phi / L^{2}$. On the $2 \mathrm{~d}$ torus $T_{12}$, the $4+1 \mathrm{~d}$ CS term is reduced to $S_{\text {eff }}=$ $\frac{\phi}{2 \pi} \frac{C_{2}}{4 \pi} \int d x_{1} d x_{2} d x_{0} \epsilon^{\mu \nu \rho} A_{\mu} \partial_{v} A_{\rho}=\frac{C_{2}}{4 \pi} \int d x_{1} d x_{2} d x_{0} \epsilon^{\mu \nu \rho} A_{\mu} \partial_{v} A_{\rho}$ with $\mu, v, \rho=0,1,2$. This is just a $2+1 \mathrm{~d}$ CS term. We consider an adiabatic evolution of gauge potential $\left(A_{1}(t), A_{2}(t)\right)$ along a rectangular path $C:(0,0) \rightarrow(2 \pi / L, 0) \rightarrow(2 \pi / L, 2 \pi / L) \rightarrow$ $(0,2 \pi / L) \rightarrow(0,0)$, and we have Berry phase given by CS term as $S_{\text {eff }}=2 \pi C_{2}$. Because $A_{i}$ is gauge equivalent to $A_{i}+2 \pi / L$, the system is actually adiabatically evolving on a torus parameterized by $\left(A_{1}, A_{2}\right), A_{1}, A_{2} \in[0,2 \pi / L]$. With the path $C$ enclosing the entire torus surface, we have the Dirac quantization condition $C_{2}=$ integer. The only assumption in this argument is that we have a unique ground state; otherwise the Berry phase is generally non-abelian[30] and our argument fails. From the integer quantization of $C_{2}$, we reach the conclusion that $C_{2}$ is unchanged when the the Hamiltonian is tuned smoothly and the energy gap remains open.

Now we turn to the topological order parameter for such $4+1 d$ TIs with general interaction. We define

$$
\begin{aligned}
N_{4} \equiv & \frac{\pi^{2}}{15} \int \frac{d^{5} k}{(2 \pi)^{5}} \operatorname{Tr}\left[\epsilon^{\mu \nu \rho \sigma \tau} G \partial_{\mu} G^{-1} G \partial_{\nu} G^{-1} G \partial_{\rho} G^{-1}\right. \\
& \left.\times G \partial_{\sigma} G^{-1} G \partial_{\tau} G^{-1}\right]
\end{aligned}
$$

where the partial derivative is taken with respect to the momenta $k_{\mu}=\left(k_{0}, k_{1}, k_{2}, k_{3}, k_{4}\right)$, and $G=G(k)$ is the full Green's function. From the spectral representation of $G(k)$, it can be shown that $G(k)$ is a smooth function of $k$ when the energy gap is nonzero. Under a smooth change of physical parameter, and the associated smooth change of $\delta G(k)$, the variation $\delta N_{4}$ vanishes, as proven in Eq. (C1) of Ref. [6]. Therefore, $N_{4}$ is a topological invariant. Next we show that $N_{4}$ is always an integer. Generally, $G(k)$ defines a map from the five dimensional $k$ space to the space of non-singular Green's functions, belonging to the group $\operatorname{GL}(n, \mathrm{C})$, whose homotopy group is labeled by an integer:

$$
\pi_{5}(\mathrm{GL}(n, \mathrm{C}))=\mathbb{Z}
$$

which is exactly $N_{4}$. Here $n \geq 3$ is the number of bands. Finally, we show that the identity

$$
C_{2}=N_{4}
$$

which is a $4+1 \mathrm{~d}$ analog of Eq.(3), holds for general interacting systems. To be specific, we consider a typical phase diagram shown in Fig.(2) for an interacting Hamiltonian $H=H_{0}(\lambda)+H_{1}(g)$, where $H_{0}$ is the non-interacting part including terms such as $t_{i j} c_{i}^{\dagger} c_{j}$, and $H_{1}$ is the electronelectron(e-e) interaction part including terms such as the Hubbard interaction $g n_{i \downarrow} n_{i \uparrow}$. These two parts $H_{0}(\lambda)$ and $H_{1}(g)$ are determined by single particle parameters $\lambda=\left(\lambda_{1}, \lambda_{2}, \cdots\right)$ and coupling constants $g=\left(g_{1}, g_{2}, \cdots\right)$ respectively. When $(\lambda, g)$ are smoothly tuned, the ground state evolves smoothly so long as the energy gap remains open, and therefore both $N_{4}$ and $C_{2}$ remain unchanged, as has been discussed. Only when the gap closes, the full Green's function $G$ becomes singular, and $N_{4}$ and $C_{2}$ can both change, as indicated by the curve $a b$ in Fig.(2). Arbitrarily picking a gapped state $A$ in the phase diagram, we can find a path $A C$ connecting $A$ to a non-interacting state $C$, without crossing the phase boundary $a b$. Now we have $N_{4}(A)=N_{4}(C)$ and $C_{2}(A)=C_{2}(C)$. In addition, because $C$ is a non-interacting state, $C_{2}$ can be simply calculated from a single Feynman diagram, which gives the result $C_{2}(C)=N_{4}(C)[6,31-33]$. Therefore, we have $N_{4}(A)=C_{2}(A)$ for generally interacting ground state $A$. 


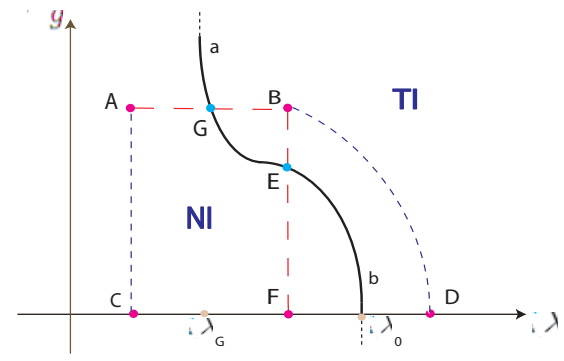

FIG. 2: Phase diagram on the $(\lambda, g)$ plane. The dark curve $a b$ is the phase boundary seperating the normal insulators(NI) and topologically nontrivial insulators(TI). The ground states are gapped everywhere except on $a b$. The true parameter space is in fact infinite dimensional, but this $2 \mathrm{~d}$ diagram can illustrate the main features. This diagram is applicable in both $4+1 \mathrm{~d}$ and $3+1 \mathrm{~d}$.

Our discussion so far can be straightforwardly generalized to CS insulators in $2 n+1 \mathrm{~d}$ for integer $n$. In particular, we give here the topological order parameter $N_{2}$ for the two dimensional quantum anomalous Hall insulator [34, 35]:

$$
N_{2}=\frac{\pi}{3} \int \frac{d^{3} k}{(2 \pi)^{3}} \operatorname{Tr}\left[\epsilon^{\mu \nu \rho} G \partial_{\mu} G^{-1} G \partial_{\nu} G^{-1} G \partial_{\rho} G^{-1}\right]
$$

This formula generalizes the TKNN formula[36] to the interacting systems, and is equivalent to the Chern number formula with twisted boundary condition[30]. It could be useful to determine the phase diagram of an quantum anomalous Hall insulator induced by interactions[19]. This formula could also be used to define the chiral topological superconductor [37], where $G$ is regarded as the Green's function of the BdG quasiparticles.

The $\mathcal{T}$-invariant TI in $3+1 d$ : Now we turn attention to $\mathcal{T}$ invariant insulators in $3+1 \mathrm{~d}$. Following the logic of dimensional reduction[6], we propose a topological order parameter defined by Eq.(1).

Now we show $P_{3}$ is quantized to be integer or half-integer. First, it can be obtained that $G(k, u=0)$ has the $\mathcal{T}$ property $G\left(k_{0},-\mathbf{k}, u=0\right)=T G\left(k_{0}, \mathbf{k}, u=0\right)^{T} T^{\dagger}$, where ${ }^{T}$ means transposition and $T$ is the time-reversal matrix satisfying $T^{\dagger} T=1$ and $T^{*} T=-1$. Given an extension $G(k, u)$, with $u \in[0,1]$, we consider a mirror extension, defined as $\tilde{G}(k, u)$, with the property $\tilde{G}\left(k_{0}, \mathbf{k}, u\right)=T G\left(k_{0},-\mathbf{k},-u\right)^{T} T^{\dagger}, u \in[-1,0]$, for which $\tilde{P}_{3}$ is defined similarly to Eq.(1), but with $u$ integrated over $[-1,0]$ instead of $[0,1]$. It can be checked that $P_{3}=\tilde{P}_{3}$ for a mirror pair of extensions $G$ and $\tilde{G}$, therefore, we have

$$
\begin{aligned}
2 P_{3}= & P_{3}+\tilde{P}_{3} \\
= & \frac{\pi}{6} \int_{-1}^{1} d u \int \frac{d^{4} k}{(2 \pi)^{4}} \operatorname{Tr} \epsilon^{\mu \nu \rho \sigma}\left[\hat{G} \partial_{\mu} \hat{G}^{-1}\right. \\
& \left.\times \hat{G} \partial_{\nu} \hat{G}^{-1} \hat{G} \partial_{\rho} \hat{G}^{-1} \hat{G} \partial_{\sigma} \hat{G}^{-1} \hat{G} \partial_{u} \hat{G}^{-1}\right] \\
= & n
\end{aligned}
$$

where $n$ is an integer, and $\hat{G}(k, u)=G(k, u)$ when $u \in[0,1]$ while $\hat{G}(k, u)=\tilde{G}(k, u)$ when $u \in[-1,0]$. The integral in Eq. (9) is an integer because $\hat{G}(k, u=1)=\hat{G}(k, u=-1)$ are both the same reference Green's function, so we can identify the manifolds $(k, u=-1)$ and $(k, u=1)$ as the same manifold, and we have an integral over a torus in $\mathbf{k} \operatorname{space}\left(k_{0}\right.$ is still integrated over $(-\infty, \infty)$ ). From Eq. (9), we know that $P_{3}=n / 2$ is quantized to be integer or half-integer. Therefore, its variation under a infinitesimal change of $G$ vanishes, just like $N_{4}$. It is well known that the WZW terms have integer ambiguity[29]. If we choose two different WZW extensions, their difference is generally given by the homotopy class in Eq. (6), which is an integer. The integer ambiguity of $P_{3}$ translates into the periodicity of $\theta$ under a shift of $2 \pi$, by the identification of Eq. (3), which can be obtained in similar way to Eq.(7). Therefore, $P_{3}$ is a $Z_{2}$ topological order parameter for $3+1 \mathrm{~d} \mathcal{T}$-invariant interacting insulators.

Now we briefly discuss the effect of disorder. It is convenient to consider a large but finite size insulator with periodic boundary condition. To define $P_{3}$, we can use a twisted boundary condition [30, 38] with twisted phase $\theta_{i} \in$ $[0,2 \pi], i=1,2,3$. The Green's function, as a function of $\theta_{i}$, is now a matrix whose rank is proportional to the system size instead of the number of bands. The $P_{3}$ for disordered insulator is defined by simply replacing $k_{i}$ in Eq. (1) by $\theta_{i}$. It can be shown that this new definition of $P_{3}$ reduces to Eq.(1) in the absence of disorder. The analysis in the interacting insulator applies to the disordered insulator as well, and we have similar phase diagram as Fig.(2), with $\lambda$ interpreted as the spin-orbit coupling and $g$ interpreted as the disorder strength.

Therefore, using the simple topological order parameter $P_{3}$ expressed in term of Green's function, we have a unified picture of TI in the presence of interaction and disorder. The same discussion applies to $4+1 \mathrm{~d}$ and $2+1 \mathrm{~d}$, where we have disorder-induced quantum spin Hall states, which has been studied in Ref.[39, 40].

The $\mathcal{T}$-invariant TI in $2+1 d$ : The topological order parameter in $2+1 \mathrm{~d}$ is similar to the $3+1 \mathrm{~d}$ case. The only difference is that in $2+1 \mathrm{~d}$ we need two WZW extension parameters $u$ and $v$ to define our topological order parameter (see Fig.(1b)). Given a $2+1 \mathrm{~d}$ insulating system with full Green's function $G(k)$, we can extend $G(k)$ to a $2 \mathrm{~d}$ torus parameterized by $(u, v) ; u, v \in[-1,1]$, i.e. we define $G(k, u, v)$ satisfying $G(k, 0,0)=G(k)$, and $G\left(k_{0}, \mathbf{k}, u, v\right)=T G\left(k_{0},-\mathbf{k},-u,-v\right)^{T} T^{\dagger}$. The Green's functions at the boundary $u=1$ and $v=1$ are fixed to be some reference value, which can be chosen to be trivial, say with flat bands, in the same way as in $3+1 \mathrm{~d}$. The topological order parameter in $2+1 \mathrm{~d}$ is defined as

$$
\begin{aligned}
P_{2}= & \frac{1}{120} \epsilon^{\mu \nu \rho \sigma \tau} \int_{-1}^{1} d u \int_{-1}^{1} d v \int \frac{d^{3} k}{(2 \pi)^{3}} \operatorname{Tr}\left[G \partial_{\mu} G^{-1}\right. \\
& \times G \partial_{\nu} G^{-1} G \partial_{\rho} G^{-1} G \partial_{\sigma} G^{-1} G \partial_{\tau} G^{-1} \\
= & 0 \text { or } 1 / 2 \text { (mod integer) }
\end{aligned}
$$

where $\epsilon^{\mu \nu \rho \sigma \tau}$ is the anti-symmetric tensor taking value 1 when the variables are ordered as $\left(k_{0}, k_{1}, k_{2}, u, v\right)$. The cases $P_{2}=0$ and $P_{2}=1 / 2$ modulo integer correspond to topologically trivial and nontrivial insulators in $2+1 \mathrm{~d}$, respectively. This 
topological order parameter $P_{2}$ is valid for interacting quantum spin Hall systems in $2+1 \mathrm{~d}$, including states in the Mott regime[19]. $P_{2}$ can be physically measured by the fractional charge at the edge of the quantum spin Hall state 41]. Analog of $P_{3}$ and $P_{2}$ in $(1+1) \mathrm{D}$ cannot be defined because its value (mod integer) would vary when homotopically nonequivalent WZW extensions are used.

In conclusion we have introduced topological order parameters for $\mathcal{T}$-invariant TIs in four, three and two dimensions. These topological order parameters are defined in terms of the full Green's function, and apply to both interacting and disordered systems. These order parameters take quantized values which are stable against small changes of physical parameters, and they can be measured directly in experiments. Throughout this paper, we assume no ground state degeneracy. New fractionalized topological phases can emerge when this fundamental assumption is removed [42 44].

This work is supported by the NSF under grant numbers DMR-0904264. We thank Suk Bum Chung, Joseph Maciejko, Srinivas Raghu, Ramamurti Shankar, Shao-Long Wan and Yong-shi Wu for helpful discussions. Z.W. acknowledges the support of NSF of China(Grant No.10675108), CSC and SLAC National Lab. X.L.Q acknowledges the support of Microsoft Research Station Q.

[1] X. L. Qi and S. C. Zhang, Phys. Today 63, 33 (2010).

[2] J. Moore, Nature 464, 194 (2010).

[3] M. Z. Hasan and C. L. Kane, arxiv: cond-mat/1002.3895.

[4] S. C. Zhang and J. P. Hu, Science 294, 823 (2001).

[5] B. A. Bernevig, C. H. Chern, J. P. Hu, N. Toumbas, and S. C. Zhang, Annals of Physics 300, 185 (2002).

[6] X.-L. Qi, T. Hughes, and S.-C. Zhang, Phys. Rev. B 78, 195424 (2008).

[7] A. Kitaev, arxiv: cond-mat/0901.2686.

[8] S. Ryu, A. Schnyder, A. Furusaki, and A. Ludwig, arxiv: condmat/0912.2157.

[9] C. L. Kane and E. J. Mele, Phys. Rev. Lett. 95, 146802 (2005).

[10] L. Fu, C. L. Kane, and E. J. Mele, Phys. Rev. Lett. 98, 106803 (2007).

[11] J. E. Moore and L. Balents, Phys. Rev. B 75, 121306 (2007).

[12] R. Roy, Phys. Rev. B 79, 195322 (2009).

[13] Z. Wang, X.-L. Qi, and S.-C. Zhang, arxiv: condmat/0910.5954.

[14] X.-L. Qi, R. Li, J. Zang, and S.-C. Zhang, Science 323, 1184
(2009).

[15] A. M. Essin, J. E. Moore, and D. Vanderbilt, Phys. Rev. Lett. 102, 146805 (2009).

[16] A. Karch, Phys. Rev. Lett. 103, 171601 (2009).

[17] G. Rosenberg and M. Franz, arXiv: cond-mat/1001.3179.

[18] L. Fidkowski and A. Kitaev, arxiv: cond-mat/0904.2197.

[19] S. Raghu, X.-L. Qi, C. Honerkamp, , and S.-C. Zhang, Phys. Rev. Lett. 100, 156401 (2008).

[20] A. Shitade, H. Katsura, J. Kune, X.-L. Qi, S.-C. Zhang, and N. Nagaosa, Phys. Rev. Lett. 102, 256403 (2009).

[21] D. A. Pesin and L. Balents, arxiv: cond-mat/0907.2962.

[22] Y. Zhang, Y. Ran, and A. Vishwanath, Phys. Rev. B 79, 245331 (2009).

[23] B. Seradjeh, J. E. Moore, and M. Franz, Phys. Rev. Lett. 103, 066402 (2009).

[24] R. Li, J. Wang, X.-L. Qi, and S.-C. Zhang, Nature Physics 6, 284 (2010).

[25] M. Dzero, K. Sun, V. Galitski, and P. Coleman, Phys. Rev. Lett. 104, 106408 (2010).

[26] S. Rachel and K. Le Hur, Phys. Rev. B 82, 075106 (2010).

[27] W.-K. Tse and A. H. MacDonald, arXiv:1003.2260.

[28] J. Maciejko, X.-L. Qi, H. D. Drew, and S.-C. Zhang, arXiv: cond-mat/1004.2514.

[29] E. Witten, Nucl. Phys. B 223, 422 (1983).

[30] Q. Niu, D. J. Thouless, and Y.-S. Wu, Phys. Rev. B 31, 3372 (1985).

[31] A. J. Niemi and G. W. Semenoff, Phys. Rev. Lett. 51, 2077 (1983).

[32] M. F. L. Golterman, K. Jansen, and D. B. Kaplan, Phys. Lett. B 301, 219 (1993).

[33] G. E. Volovik, JETP Lett. 75, 63 (2002).

[34] F. D. M. Haldane, Phys. Rev. Lett. 61, 2015 (1988).

[35] X.L. Qi, Y.S. Wu, and S.C. Zhang, Phys. Rev. B 74, 085308 (2006).

[36] D. J. Thouless, M. Kohmoto, M. P. Nightingale, and M. den Nijs, Phys. Rev. Lett. 49, 405 (1982).

[37] G. E. Volovik, The Universe in a Helium Droplet (Oxford University Press, USA, 2003).

[38] J. E. Avron and R. Seiler, Phys. Rev. Lett. 54, 259 (1985).

[39] J. Li, R.-L. Chu, J. K. Jain, and S.-Q. Shen, Phys. Rev. Lett. 102, 136806 (2009).

[40] C. W. Groth, M. Wimmer, A. R. Akhmerov, J. Tworzyd, and C. W. J. Beenakker, Phys. Rev. Lett. 103, 196805 (2009).

[41] X.-L. Qi, T. L. Hughes, and S.-C. Zhang, Nat. Phys. 4, 273 (2009).

[42] B.A. Bernevig and S.C. Zhang, Phys. Rev. Lett. 96, 106802 (2006).

[43] M. Levin and A. Stern, Phys. Rev. Lett. 103, 196803 (2009).

[44] J. Maciejko, X.-L. Qi, A. Karch and S. C. Zhang, arXiv: 1004.3628. 